\title{
A Genetic Screen Reveals Novel Targets to Render Pseudomonas aeruginosa Sensitive to Lysozyme and Cell Wall-Targeting Antibiotics
}

\author{
Kang-Mu Lee ${ }^{1}$, Keehoon Lee ${ }^{1,2}$, Junhyeok Go ${ }^{1,2}$, In Ho Park ${ }^{1}$, Jeon-Soo Shin 1, 2,3,4, \\ Jae Young Choi ${ }^{2,5}$, Hyun Jik Kim ${ }^{6}$ and Sang Sun Yoon ${ }^{1,2,4 *}$ \\ ${ }^{1}$ Department of Microbiology and Immunology, Yonsei University College of Medicine, Seoul, South Korea, ${ }^{2}$ Brain Korea 21 \\ PLUS Project for Medical Sciences, Yonsei University College of Medicine, Seoul, South Korea, ${ }^{3}$ Department of Microbiology \\ and Immunology, Severance Biomedical Science Institute, Yonsei University College of Medicine, Seoul, South Korea, \\ ${ }^{4}$ Department of Microbiology and Immunology, Institute for Immunology and Immunological Diseases, Yonsei University \\ College of Medicine, Seoul, South Korea, ${ }^{5}$ Department of Otorhinolaryngology, Yonsei University College of Medicine, Seoul, \\ South Korea, ${ }^{6}$ Department of Otorhinolaryngology, Seoul National University College of Medicine, Seoul, South Korea
}

OPEN ACCESS

Edited by:

D. Scott Merrell,

Uniformed Services University of the

Health Sciences, USA

Reviewed by:

Alison Criss,

University of Virginia, USA Amanda G. Oglesby-Sherrouse,

University of Maryland, USA

*Correspondence:

Sang Sun Yoon sangsun_yoon@yuhs.ac

Received: 01 November 2016 Accepted: 13 February 2017 Published: 01 March 2017

Citation:

Lee K-M, Lee K, Go J, Park IH,

Shin J-S, Choi JY, Kim HJ and Yoon SS (2017) A Genetic Screen Reveals Novel Targets to Render Pseudomonas aeruginosa Sensitive to Lysozyme and Cell Wall-Targeting Antibiotics.

Front. Cell. Infect. Microbiol. 7:59. doi: 10.3389/fcimb.2017.00059
Pseudomonas aeruginosa is capable of establishing airway infections. Human airway mucus contains a large amount of lysozyme, which hydrolyzes bacterial cell walls. $P$. aeruginosa, however, is known to be resistant to lysozyme. Here, we performed a genetic screen using a mutant library of PAO1, a prototype $P$. aeruginosa strain, and identified two mutants $(\triangle b a m B$ and $\triangle f a b Y$ ) that exhibited decrease in survival after lysozyme treatment. The bamB and fabY genes encode an outer membrane assembly protein and a fatty acid synthesis enzyme, respectively. These two mutants displayed retarded growth in the airway mucus secretion (AMS). In addition, these mutants exhibited reduced virulence and compromised survival fitness in two different in vivo infection models. The mutants also showed susceptibility to several antibiotics. Especially, $\triangle$ bamB mutant was very sensitive to vancomycin, ampicillin, and ceftazidime that target cell wall synthesis. The $\Delta$ fabY displayed compromised membrane integrity. In conclusion, this study uncovered a common aspect of two different $P$. aeruginosa mutants with pleiotropic phenotypes, and suggests that BamB and FabY could be novel potential drug targets for the treatment of $P$. aeruginosa infection.

Keywords: Pseudomonas aeruginosa, lysozyme, treatment regimen, airway infection, multi-drug resistance

\section{INTRODUCTION}

Antibiotic-resistant $P$. aeruginosa strains are emerging at a significantly faster rate than the introduction of new anti-Pseudomonal agents (Pendleton et al., 2013). Furthermore, prolonged use of antibiotics has been proven to be a critical risk factor in the selection of multi-drug resistant variants, especially in the case of $P$. aeruginosa infection (Merlo et al., 2007). It is, therefore, crucial to devise a revolutionary strategy to combat $P$. aeruginosa infection. One such way would be to take advantage of the host innate defense molecules. Since the early discovery of the inherent lysozyme resistance of $P$. aeruginosa (Warren et al., 1955), no significant efforts have been made to increase the susceptibility of $P$. aeruginosa to lysozyme.

Lysozyme is an important antibacterial protein that is abundantly present in the human airway (Duszyk, 2001; Dubin et al., 2004; Dajani et al., 2005). Its enzymatic activity is to 
cleave $\beta$-1,4-glycosidic linkages on the $\mathrm{N}$-acetylglucosamine (NAG) and N-acetylmuramic acid (NAM) polysaccharide chains within the bacterial peptidoglycan layer (Vanderwinkel et al., 1995). Lysozyme found in the human airway is more active than egg-white lysozyme due to distinct structural differences between the two molecules (Marx et al., 1986). Although lysozyme has been proposed to play a crucial role in host defense, persistent $P$. aeruginosa airway infection is a major cause of morbidity and mortality in cystic fibrosis (CF) patients. Moreover, higher lysozyme activity has been detected in the serum and saliva of CF patients compared with normal individuals (Hughes et al., 1982). Likewise, lysozyme activity was also higher in bronchoalveolar lavage fluid (BALF) collected from CF patients (Sagel et al., 2009). Together, these results suggest that human lysozyme is ineffective in killing $P$. aeruginosa during infection. The lack of lysozyme effectiveness against $P$. aeruginosa has also been attributed to its active production of elastase, a major metalloprotease that can cleave human lysozyme (Jacquot et al., 1985).

Besides the infection in CF patients, $P$. aeruginosa is one of the major pathogens that cause ventilator-associated pneumonia (VAP). Especially, the pneumonia and sepsis by P. aeruginosa are the serious threats to the cardiac or thoracic surgical patients and trauma patients in the Intensive Care Unit (ICU; Berra et al., 2010). P. aeruginosa can also cause acute lung injury by injecting the ExoU toxin into the cytosol of eukaryotic cells using Type III Secretion System (TTSS; Pankhaniya et al., 2004). The acute $P$. aeruginosa infection could disrupt the alveolar epithelial barrier to promote necrosis of lung epithelial cells and sepsis, leading rapid increase in mortality (Sawa, 2014).

In this study, we aimed to identify novel interventional approaches that increase the sensitivity of $P$. aeruginosa to lysozyme. To this end, we isolated $P$. aeruginosa mutants that lost their viability when treated with lysozyme and sought to describe the genetic and phenotypic bases of their lysozyme sensitivity. Such defective mutants were found to be incapable of propagating in airway mucus secretions (AMSs) or establishing infection in the mouse airway, two locations where lysozyme is present in high concentrations. Results provided here will stimulate future works to propose novel approaches for inhibiting $P$. aeruginosa growth under lysozymerich environments.

\section{MATERIALS AND METHODS}

\section{Experimental Ethics}

Experiments using human subjects and experimental animals were performed in strict accordance with guidelines provided by Yonsei University. Protocols were reviewed and approved by Institutional Review Board of Yonsei University College of Medicine. Permit numbers for primary culture of human tissues and mouse infection experiment were 2014-1842-001 and 20130369-5, respectively.

\section{Bacterial Strains and Growth Conditions}

A prototype strain of $P$. aeruginosa called PAO1 was used in this study (Yoon et al., 2006). The bacterial strains of the other species, as listed in Figure 1, were obtained from our laboratory

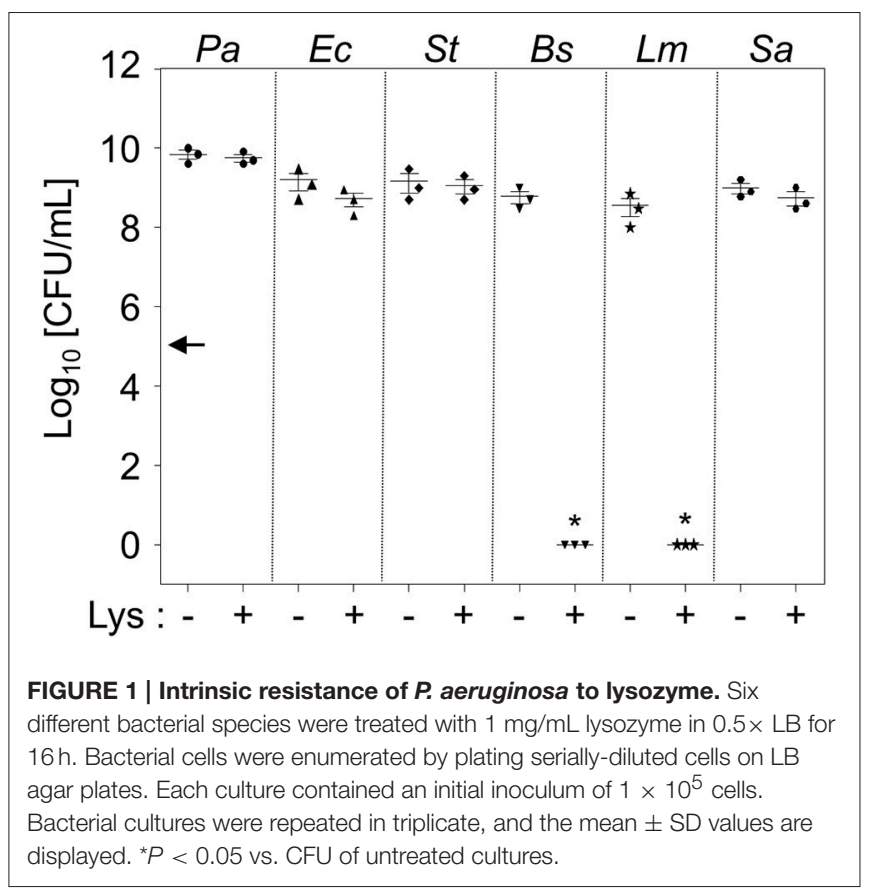

stock and were previously reported (Gi et al., 2015). Bacterial cultures were grown in Luria-Bertani medium (LB; $10 \mathrm{~g}$ tryptone, $5 \mathrm{~g}$ yeast extract, and $10 \mathrm{~g} \mathrm{NaCl} / \mathrm{l}$ ), unless otherwise stated. Bacterial cells were cultured at $37^{\circ} \mathrm{C}$ for $16 \mathrm{~h}$ and shaken at $200 \mathrm{rpm}$. Bacterial growth in AMS and survival following the treatments with diverse antibiotics were assessed by quantifying colony forming units (CFUs).

\section{Mutant Library Screen for Lysozyme-Sensitive Mutants and Preparation of Airway Mucus Secretion (AMS)}

A transposon (Tn) insertion mutant library of PAO1 was constructed following the procedures described elsewhere (Lee et al., 2016). In brief, PAO1 was conjugated with Escherichia coli SM10/ $\lambda$ pir harboring the pBTK30 plasmid (Table 1). Gentamicin $(\mathrm{Gm})$-resistant transconjugants were grown on LB agar plates containing $50 \mu \mathrm{g} / \mathrm{ml} \mathrm{Gm}$ and $50 \mu \mathrm{g} / \mathrm{ml}$ Irgasan (Sigma). Irgasan was added to eliminate E. coli donor cells. To isolate lysozyme-sensitive mutants, each individual mutant was grown in $0.5 \times \mathrm{LB}$ media $(50 \% \mathrm{LB}+50 \%$ distilled water) containing $1 \mathrm{mg} / \mathrm{mL}$ lysozyme (Sigma) in 96 well plates. Bacterial growth was monitored by measuring the $\mathrm{OD}_{600}$. The Tn insertion site of each defective mutant was determined by arbitrary PCR, followed by DNA sequencing (Lee et al., 2016). Preparation of AMS samples from Normal Healthy Trachea Epithelial (NHTE) cells and bacterial growth in AMS were conducted as previously described (Gi et al., 2015).

\section{Construction of the PA0420, PA3800, and PA5174 Clean Deletion Mutants}

The bioA, bamB, and fabY deletion mutants were created by allelic replacement as previously described (Lee et al., 2012). 
TABLE 1 | Bacterial strains and plasmids used in this study.

\begin{tabular}{|c|c|c|}
\hline Strains or plasmids & Relevant characteristics & References or source \\
\hline \multicolumn{3}{|l|}{$P$. aeruginosa strains } \\
\hline PAO1 & Standard lab strain & Yoon et al., 2006 \\
\hline$\triangle P A 0420$ & PA01, PA0420 gene deleted & This study \\
\hline$\triangle P A 3800$ & PAO1, PA3800 gene deleted & This study \\
\hline$\triangle P A 5174$ & PAO1, PA5174 gene deleted & This study \\
\hline$\triangle P A 3800 / p J N 105$ & $\triangle P A 3800$ harboring pJN105 & This study \\
\hline$\triangle P A 5174 / p J N 105$ & $\triangle P A 5174$ harboring pJN105 & This study \\
\hline$\triangle P A 3800 /$ pJN105::PA3800 & $\triangle P A 3800$ harboring pJN105::PA3800 & This study \\
\hline$\triangle P A 5174 /$ pJN105::PA5174 & $\triangle P A 5174$ harboring pJN105::PA5174 & This study \\
\hline \multicolumn{3}{|l|}{ E. coli strains } \\
\hline 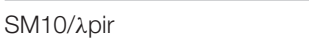 & $\mathrm{Km}^{\mathrm{r}}$ thi-1 thr leu tonA lacY supE recA::RP4-2-Tc::Mu pir ${ }^{+}$, for conjugal transfer & Simon et al., 1983 \\
\hline \multicolumn{3}{|c|}{ OTHER BACTERIAL SPECIES } \\
\hline Staphylococcus aureus & ATCC 29213 & Lab collection \\
\hline Bacillus subtilis & ATCC 6633 & Lab collection \\
\hline Escherichia coli & ATCC 25922 & Lab collection \\
\hline Salmonella typhimurium & LT2 strain & Lab collection \\
\hline Listeria monocytogenes & ATCC 19111 & Lab collection \\
\hline \multicolumn{3}{|l|}{ PLASMIDS } \\
\hline pBTK30 & Transposon vector for construction of a random mutant library, $\mathrm{Gm}^{r}$ & Kim et al., 2012 \\
\hline pCVD442 & sacB suicide vector derived from plasmid pUM24 & Lee et al., 2012 \\
\hline pJN105 & araC-PBAD cassette cloned in pBBR1MCS-5, Gm & Newman and Fuqua, 1999 \\
\hline pJN105::PA3800 & pJN105 with wild-type copy of $P A 3800$ gene under arabinose-inducible promoter & This study \\
\hline pJN105::PA5174 & pJN105 with wild-type copy of PA5174 gene under arabinose-inducible promoter & This study \\
\hline
\end{tabular}

Briefly, flanking sequences ( $\sim 600 \mathrm{bp})$ at both ends of each gene were PCR amplified with primers listed in Table 2. Two inner primers (upstream reverse primer and downstream forward primer) are complementary to each other. In this strategy, the $3^{\prime}$ end of the upstream sequence and the $5^{\prime}$-end of the downstream sequence are annealed during PCR amplification without further treatment. The deletion of the PA0420, PA3800, and PA5174 genes was confirmed by PCR and DNA sequencing.

\section{Genetic Complementation of the $\triangle P A 3800$ and $\triangle P A 5174$ Mutants}

To complement the $\triangle P A 3800$ and $\triangle P A 5174$, DNA fragments containing the entire $P A 3800$ and $P A 5174$ genes were amplified from the PAO1 genome and ligated into EcoRI/XbaI-treated pJN105. The resultant plasmid and the control empty plasmid (i.e., pJN105) were transferred into the $\triangle P A 3800$ and $\triangle P A 5174$ mutant by electroporation, respectively. Expression of $P A 3800$ or $P A 5174$ gene is induced by $0.01 \% \mathrm{~L}$-arabinose (Sigma).

\section{Bacterial Growth in Airway Mucus Secretion (AMS)}

Bacterial cultures grown in LB for $8 \mathrm{~h}$ were diluted in PBS to get bacterial suspensions with the $10^{5} \mathrm{CFU} / \mathrm{mL}$. Ten microliters of each diluent was inoculated into $100 \mu \mathrm{L}$ AMS to achieve the initial inoculum size of $\sim 10^{3} \mathrm{CFU}$ in AMS. Bacterial cells were then grown for $16 \mathrm{~h}$ in a humidified $37^{\circ} \mathrm{C}$ incubator. Bacterial growth was assessed by measuring the growth index. The values for [CFU after $16 \mathrm{~h}$ in AMS / CFU after $0 \mathrm{~h}$ in AMS] were calculated and plotted as the growth index.

\section{Mouse Infection and Caenorhabditis elegans Survival Test}

Animal experiments were approved by the Committee on the Ethics of Animal Experiments of Yonsei University College of Medicine (IACUC permit number: 2013-0369-5). Animal experiments were conducted following national guidelines provided by the Korean government (Ministry for Food, Agriculture, Forestry and Fisheries) and in strict accordance with the institutional guidelines for animal care and use of laboratory animals. To test the virulence of the $\triangle b a m B$ and $\Delta f a b Y$ mutants in vivo, 8-week-old C57BL/6N inbred female mice (Orient, Korea) were intranasally infected with $5 \times 10^{6}$ cells of each strain $(n=4)$. The lungs of the infected mice were removed at $16 \mathrm{~h}$ post-infection. The harvested lungs were homogenized, and the number of bacteria in each organ was measured by quantifying CFUs. Caenorhabditis elegans survival tests were performed as described previously (Go et al., 2014).

\section{Electron Microscope}

Lysozyme-treated bacterial cells were visualized using a scanning electron microscope (SEM), as described previously (Yoon et al., 2011). Briefly, fixed bacterial suspensions were stained with $1 \%$ OsO4 (Sigma) and then coated with gold via an ion sputter (IB3 Eiko, Japan). SEM (FE SEM S-800, Hitachi, Japan) was used at 
TABLE 2 | Primers used in this study.

\begin{tabular}{|c|c|c|c|}
\hline Gene name & Direction & Primer sequence $\left(5^{\prime}-3^{\prime}\right)^{a}$ & Restriction enzymes \\
\hline \multicolumn{4}{|l|}{ MUTANT CONSTRUCTION } \\
\hline PA0420 left & Forward & ACCTTGAGCTCGCCGAGAGATCGTCCAGGGT & Sacl \\
\hline PA0420 left & Reverse & TCCGGGTGGAAGTCGCTGACAAGGCCCATGGGCTGTCTCC & \\
\hline PA0420 right & Forward & GGAGACAGCCCATGGGCCTTGTCAGCGACTTCCACCCGGA & \\
\hline PA0420 right & Reverse & TAGAGGAGCTCAGGGCCAGAGGCGGATGGAT & $\mathrm{Sacl}$ \\
\hline PA3800 left & Forward & ACCTTGCATGCTGGTGGCAGCGCAACGGCAA & Sphl \\
\hline PA3800 left & Reverse & ACGAGCTTGCCACCGTTGCCCACCATCTCAGGCCTCTCCC & \\
\hline PA3800 right & Forward & GGGAGAGGCCTGAGATGGTGGGCAACGGTGGCAAGCTCGT & \\
\hline PA3800 right & Reverse & TAGAGGCATGCGATCCCCAGGGCTTCCTGCA & Sphl \\
\hline PA5174 left & Forward & ACCTTGAGCTCGTCCAGGCCGCCATCGAGTT & Sacl \\
\hline PA5174 left & Reverse & GGCGCTCAGTCGAGCATGTCGCCTGCTGCGTTGTAGCCAC & \\
\hline PA5174 right & Forward & GTGGCTACAACGCAGCAGGCGACATGCTCGACTGAGCGCC & \\
\hline PA5174 right & Reverse & TAGAGGAGCTCCCGGTAGCGAGGAGTTCACC & Sacl \\
\hline \multicolumn{4}{|l|}{ COMPLEMENTATION } \\
\hline \multirow[t]{2}{*}{ PA3800 complementation (cloning to pJN105) } & Forward & AATTCGAATTCAAGGAGATATACATATGGTGCAATGGAAACACGC & EcoRl \\
\hline & Reverse & ATATCTCTAGACTAGCGGATGGTGTAGGCGA & Xbal \\
\hline \multirow[t]{2}{*}{ PA5174 complementation (cloning to pJN105) } & Forward & AATTCGAATTCAAGGAGATATACATATGTCTCGACTACCGGTCATT & ECORI \\
\hline & Reverse & ATATCTCTAGATCAGTCGAGCATGTCGCTGA & Xbal \\
\hline
\end{tabular}

${ }^{a}$ Restriction enzyme recognition sequences are underlined.

an acceleration voltage of $20 \mathrm{kV}$. All images were processed using ESCAN 4000 software (Bummi Universe Co., Ltd., Seoul, Korea).

\section{Membrane Integrity Assays}

Bacterial resistance against hypo-osmotic stress was measured by monitoring the decrease of $\mathrm{OD}_{600}$ values. Each bacterial strain, grown to mid-exponential phase was harvested and washed with $1 \times$ PBS. The bacterial cells were resuspended in sterilized deionized water. The $\mathrm{OD}_{600}$ of each bacterial suspension was measured every $10 \mathrm{~min}$ for $60 \mathrm{~min}$. The data were normalized with their initial $\mathrm{OD}_{600}$-values. The PAO1, $\Delta b a m B$, and $\Delta f a b Y$ mutants were also observed under confocal laser scanning microscope (CLSM) with LIVE/DEAD ${ }^{\circledR}$ BacLight ${ }^{\mathrm{TM}}$ Bacterial Viability Kit to detect the difference in membrane integrity among these bacterial strains.

\section{Statistical Analysis}

The experiments were repeated at least in triplicate, and data are expressed as mean \pm standard deviation $(S D)$. Data were analyzed using unpaired Student's $t$-test, unless otherwise stated and $P<0.05$ were considered to be statistically significant. Logrank test was used to provide statistical significance in the $C$. elegans lifespan experiments.

\section{RESULTS}

\section{P. aeruginosa Is Highly Resistant to Lysozyme}

Prior literature demonstrated that $P$. aeruginosa can become lysozyme-sensitive only when co-treated with EDTA at $\mathrm{pH} 8.0$ (Voss, 1964) or pretreated with acetone or heat (Warren et al., 1955). As expected, PAO1 grew unencumbered when grown in
LB media supplemented with $1 \mathrm{mg} / \mathrm{mL}$ lysozyme (Figure 1). Likewise, two other Gram-negative species, Escherichia coli (Ec) and Salmonella enterica serovar Typhimurium (St), also grew normally in these conditions (Figure 1). These results further support our notion that Gram-negative bacterial cells are intrinsically resistant to lysozyme because their outer membrane prevents lysozyme from accessing the peptidoglycan in the periplasm. On the other hand, two Gram-positive species, Bacillus subtilis (Bs) and Listeria monocytogenes $(\mathrm{Lm})$, lost their viability in response to the same treatment (Figure 1). Consistent with previous reports (Bera et al., 2005, 2006), Staphylococcus aureus $(\mathrm{Sa})$ was completely resistant to the lysozyme treatment (Figure 1). PAO1 growth was not affected, even in the presence of $8 \mathrm{mg} / \mathrm{mL}$ lysozyme (data not shown), demonstrating an exceptionally high resistance to lysozyme.

\section{Dentification of Lysozyme-Sensitive PAO1 Mutants}

In order to devise a better strategy for $P$. aeruginosa infection control, it would be desirable to identify lysozyme-sensitive mutants. We therefore constructed a Tn insertion mutant library and screened it for mutants that had lost their resistance to lysozyme treatment. Since lysozyme is more active in environments with reduced ionic strength (Sorrentino et al., 1982; Verhamme et al., 1988), bacterial growth was tested in two-fold diluted LB media (termed $0.5 \times$ LB). Approximately 5,500 mutants were screened, three of which were determined to lack lysozyme resistance. In each mutant, the transposon insertion occurred in PA0420, PA3800, or PA5174 gene. To further verify the effects of these gene disruptions, we generated an in-frame deletion of each gene and examined cell growth in the presence of lysozyme. In $0.5 \times \mathrm{LB}, \triangle P A 3800$ and $\triangle P A 5174$ 
mutants exhibited significant growth inhibition in the presence of $1.0 \mathrm{mg} / \mathrm{mL}$ lysozyme (Figure $2 \mathrm{~A}$ ). After $16 \mathrm{~h}$ of growth at $37^{\circ} \mathrm{C}$ with lysozyme, the CFUs of these two mutants remained similar to those at the time of inoculum (indicated by an arrow on the y-axis), indicating that bacterial growth was prevented by lysozyme at a physiological concentration. PA3800 gene encode $\beta$-barrel assembly machinery protein $\mathrm{B}(\mathrm{BamB})$ involved in outer membrane protein assembly (Jansen et al., 2012). PA5174 gene encodes probable beta-ketoacyl synthase, which plays a role in fatty acid biosynthesis (Yuan et al., 2012; Figure 2B). The deletion of $P A 3800$ gene did not result in any growth inhibition, whereas the $\Delta f a b Y$ mutant exhibited a slightly affected growth, yielding $\sim 10$-fold less viable cell counts after $16 \mathrm{~h}$ growth in 0.5 $\times$ LB media (Figure 2A). When these mutants were grown with lysozyme, bacterial cells with crumbled rod-shaped morphology were observed (Figure S1), further demonstrating that lysozyme, an enzyme that degrades peptidoglycan polymers, damages the bacterial cell wall structure.

$P A 0420$, bioA gene, produces adenosylmethionine-8-amino7-oxononanoate aminotransferase, which is responsible for biotin biosynthesis (Beaume et al., 2015). Of note, the $\Delta$ bioA mutant exhibited inactive growth, even in the absence of lysozyme treatment, indicating that an interruption in biotin

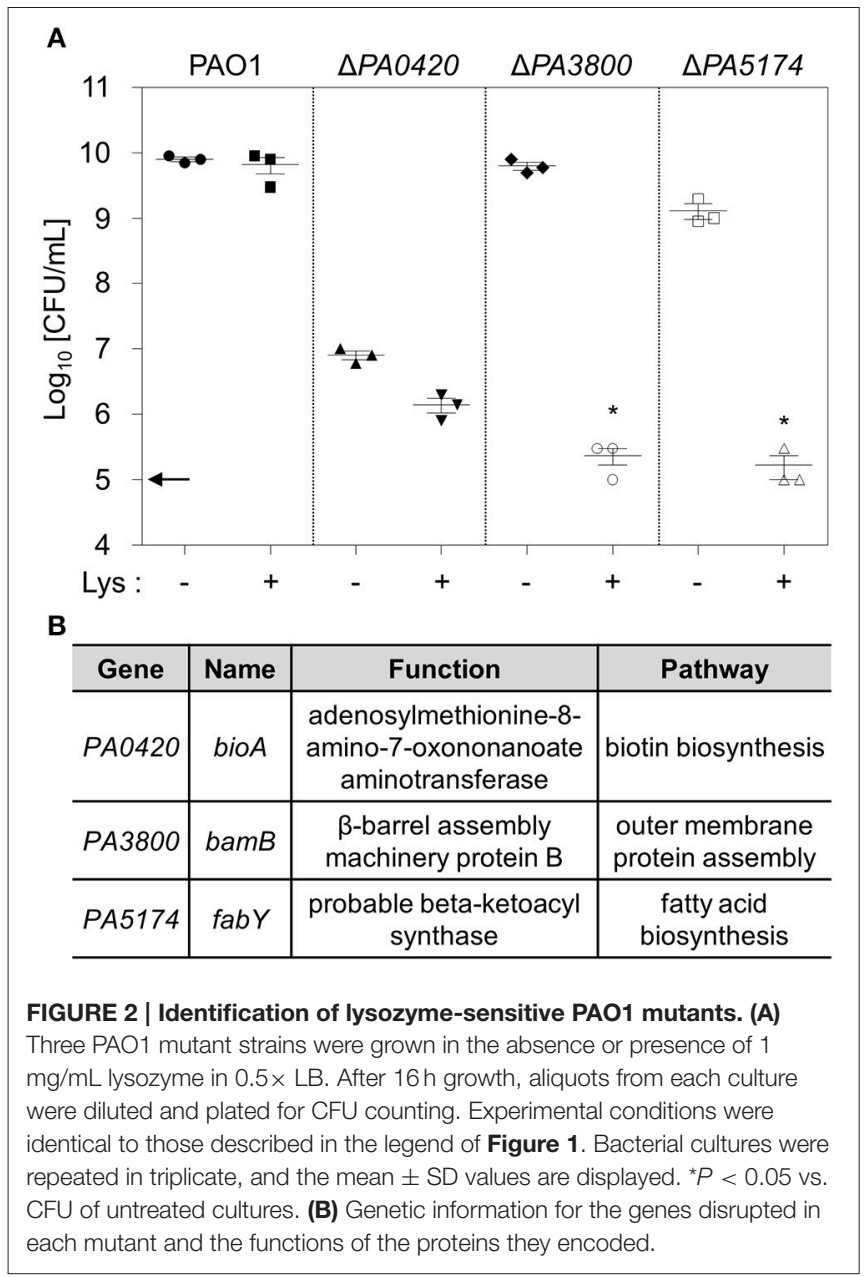

synthesis leads to defective growth in $P$. aeruginosa. When the mutant was grown in media supplemented with extraneous biotin, its growth and lysozyme susceptibility were completely restored (Figure $\mathrm{S} 2$ ). This result suggests that lysozyme sensitivity of the $\triangle b i o A$ mutant is likely associated with its faulty growth; therefore, we did not pursue any further investigation on this particular mutant.

When wild type copy of bamB or fabY gene was expressed by the arabinose-inducible promoter, each mutant became fully resistant to lysozyme treatment (Figure 3). These results further confirm that the lysozyme sensitivities observed in the mutants are indeed caused by the deletion of $b a m B$ or $f a b Y$ gene.

\section{Lysozyme-Sensitive Mutants Are also Susceptible to Treatment with Airway Mucus Secretion}

Airway mucus secretion (AMS) plays a role in the host innate defense and contains various antimicrobial components including lysozyme ( $\mathrm{Gi}$ et al., 2015). To examine bacterial response to AMS, we treated the bacterial strains with AMS collected from primary cultures of three different human tracheal tissues. In each treatment, $10^{3}$ bacterial cells were inoculated and incubated for $16 \mathrm{~h}$. After the treatments, the number of PAO1 cells increased to $\sim 5 \times 10^{6}$ cells (growth index of

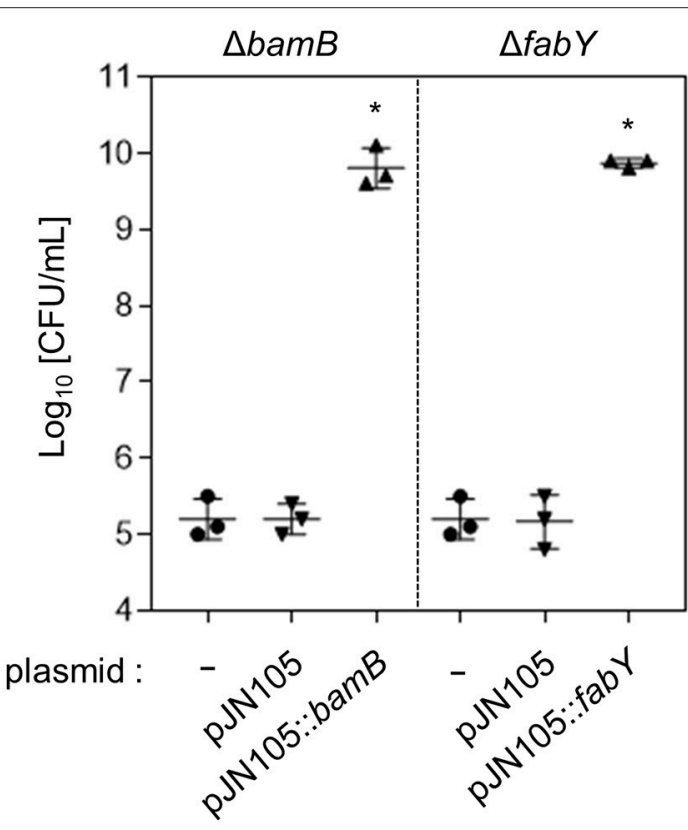

FIGURE 3 | Restored lysozyme resistance of genetically complemented mutants. The $\triangle$ bamB and $\triangle$ fabY mutants were transformed with pJN105::bamB or pJN105::fabY plasmid, respectively. As a control, each mutant was also transformed with the empty plasmid, pJN105. Bacterial strains were grown in the presence of $1 \mathrm{mg} / \mathrm{mL}$ lysozyme for $16 \mathrm{~h}$. To induce the gene expression, $0.01 \% \mathrm{~L}$-arabinose was also added in each media. Aliquots of each culture were diluted and plated for CFU quantification. Bacterial cultures were performed in triplicate, and the mean $\pm \mathrm{SD}$ values are displayed. ${ }^{*} P<0.05$ vs. CFU of each mutant harboring pJN105. 
$\sim 5,000$ ), demonstrating the capability of wild type $P$. aeruginosa to propagate in AMS (Figure 4). The growth of the $\triangle b a m B$ mutant was not as robust as that of PAO1. The average growth index of the mutant in AMS was $\sim 68$ (Figure 4). Of note, the growth of the $\triangle f a b Y$ mutant was more severely affected, yielding the growth index of $\sim 1.0$. Together, these results suggest that two lysozyme-sensitive mutants are less capable of proliferating in AMS, a frontline substance that $P$. aeruginosa encounters during the early stage of airway infection.

\section{In vivo Infectivity of the Lysozyme-Sensitive Mutants was Ameliorated}

We next examined whether these mutants were also defective in establishing infections in vivo. To address this question, we utilized two different animal infection models: mouse and nematode. First, mice were intranasally infected with bacterial cells. At $16 \mathrm{~h}$ post-infection, mouse lung homogenates were prepared, and the bacterial cells were enumerated. Increased numbers of PAO1 cells were recovered following the $16 \mathrm{~h}$ infection (Figure 5A), demonstrating that wild type $P$. aeruginosa can replicate inside the mouse airway. By comparison, decreased numbers of $\triangle b a m B$ and $\Delta f a b Y$ mutant cells were recovered, indicating a compromise in their ability to multiply inside a host airway (Figure 5A).

C. elegans is a nematode that has been widely used to study host-microbe interactions (Go et al., 2014). Furthermore, C. elegans is known to express 15 homologs of lysozyme that act as digestive enzymes for bacterial prey and, therefore, as key players for innate immunity (Mallo et al., 2002; Boehnisch et al., 2011). Given these characteristics, we postulated that

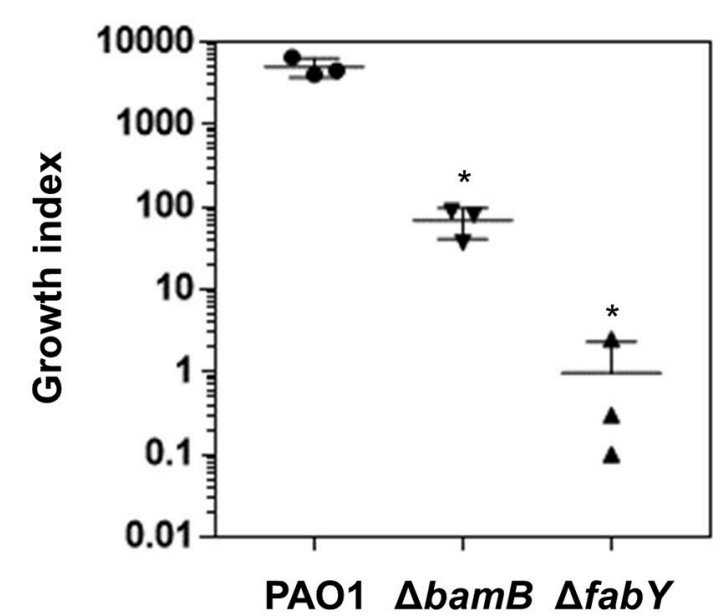

FIGURE 4 | Bacterial growth in airway mucus secretion (AMS) prepared from primary culture of human tracheal tissues. The wild type PAO1 and its two mutants, $\triangle b a m B$ and $\triangle f a b Y$, were grown in three separate AMS preparations for $16 \mathrm{~h}$. Aliquots of each culture were diluted and plated for CFU quantification. The values for CFU in16h / CFU inoculum were calculated and plotted as the growth index. Bacterial cultures were performed in triplicate, and the mean \pm SD values are displayed. ${ }^{*} P<0.05$ vs. CFU of PAO1 cells.
C. elegans could serve as an appropriate model to study in vivo infectivity of lysozyme-sensitive mutants. As shown in Figure 5B, C. elegans lived significantly longer when fed $\triangle$ bamB and, to a greater extent, $\triangle f a b Y$ mutants; the average lifespans were 9.2 \pm 0.5 (days) and $11.6 \pm 0.6$ (days), respectively. As expected, a considerably shorter lifespan (6.6 \pm 0.3 days) was observed in worms grown by feeding on PAO1 cells (Figure 5B). Together, these results demonstrate that lysozyme-sensitive mutants are also less capable of establishing in vivo virulence.

\section{Bacterial Cell Membrane Integrity was Mildly and Severely Affected in $\triangle$ bamB and $\Delta f a b Y$ Mutants, Respectively}

Because lysozyme targets bacterial peptidoglycan, we postulated that the increased lysozyme sensitivity of the mutants may be associated with altered bacterial cell membrane integrity. To address this issue, we analyzed how the lysozyme-sensitive mutants responded to hypo-osmotic stress. We also assessed the penetration of propidium iodide, a fluorescent dye that normally impermeable of healthy bacterial membrane, into bacterial cells of each strain. When PBS-washed bacterial cells were resuspended in distilled water, a marked and persistent drop in $\mathrm{OD}_{600}$-values were observed in the $\Delta f a b Y$ mutant over $1 \mathrm{~h}$ time period (Figure 6A). In contrast, bacterial cell density monitored by measuring $\mathrm{OD}_{600}$-values was decreased only in the later stage of the experiment for the case of the $\triangle b a m B$ mutant (Figure 6A). Consistent with the hypo-osmotic resistance result, $\Delta f a b Y$ mutant cells were more permeable to propidium iodide, a red fluorescent dye that only stains bacterial nucleic acids when the membrane integrity was compromised. Together, these results demonstrate that bacterial membrane integrity is affected, especially in the $\Delta f a b Y$ mutant and such a defect likely accounts for the enhanced sensitivity (i) to the AMS treatment (Figure 4) and (ii) inside the C. elegans (Figure 5B).

\section{$\Delta$ bamB Mutant Is Extremely Sensitive to Antibiotics that Target Cell Wall Synthesis}

We then explored whether lysozyme-sensitive mutants also exhibited elevated sensitivity to antibiotic treatments. To examine bacterial responsiveness to antibiotics of diverse classes, we tested vancomycin (Day et al., 1993), ceftazidime (O'Callaghan, 1986), ampicillin (Ghobashy and Chiori, 1984), tobramycin (Hoff et al., 1974), and ciprofloxacin (Roy et al., 1983), the first three of which target cell wall synthesis. Tobramycin and ciprofloxacin inhibit protein synthesis and DNA replication, respectively. At the concentrations tested, PAO1 was either unaffected or only mildly affected (Figures 7A-E). Importantly, the $\triangle b a m B$ mutant completely lost its viability in response to treatment with vancomycin, ceftazidime, and ampicillin (Figures 7A-C). The viability of the $\Delta f a b Y$ mutant was also decreased about $100 \sim 1,000$ fold in response to the same treatment (Figures 7A-C). The degree of growth inhibition in these two mutants was not as great when treated with tobramycin and ciprofloxacin (Figures 7D,E). Collectively, these results suggest that (i) the $\triangle$ bamB mutant is exceptionally sensitive to 

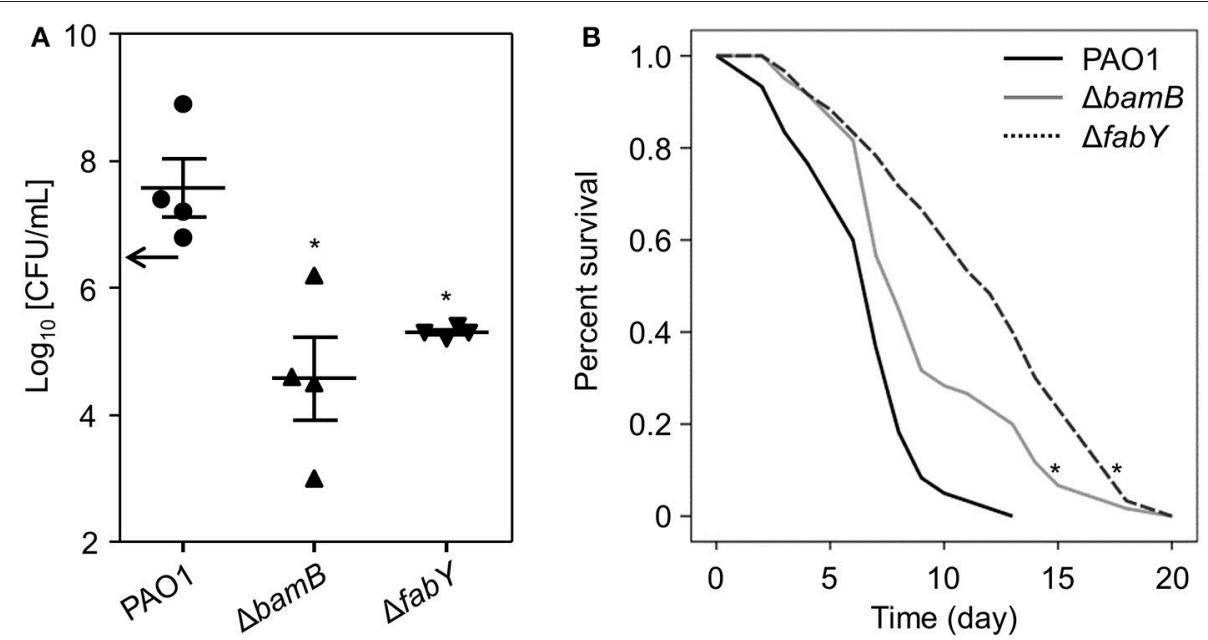

FIGURE 5 | Survival fitness and virulence of $\mathbf{\Delta} \boldsymbol{b a m B}$ and $\boldsymbol{\Delta} \boldsymbol{f a b} \boldsymbol{Y}$ mutants in vivo. (A) Bacterial counts recovered from the left lung lobes. The infection dose was $5.0 \times 10^{6}$ cells per mouse (black arrow on the $y$-axis). Mice were infected with the indicated bacterial strains for $16 \mathrm{~h} .{ }^{\star} P<0.05$ vs. CFU of PAO1 cells. (B) Survival curves of the $C$. elegans N2 strain fed with PAO1 (blue), $\Delta$ bamB (green), and $\Delta$ fabY (black). ${ }^{\star} P<0.05$ vs. the survival rate of the PAO1-fed worms. In each group, 20 worms were used. Statistical significance was determined by log-rank analysis.

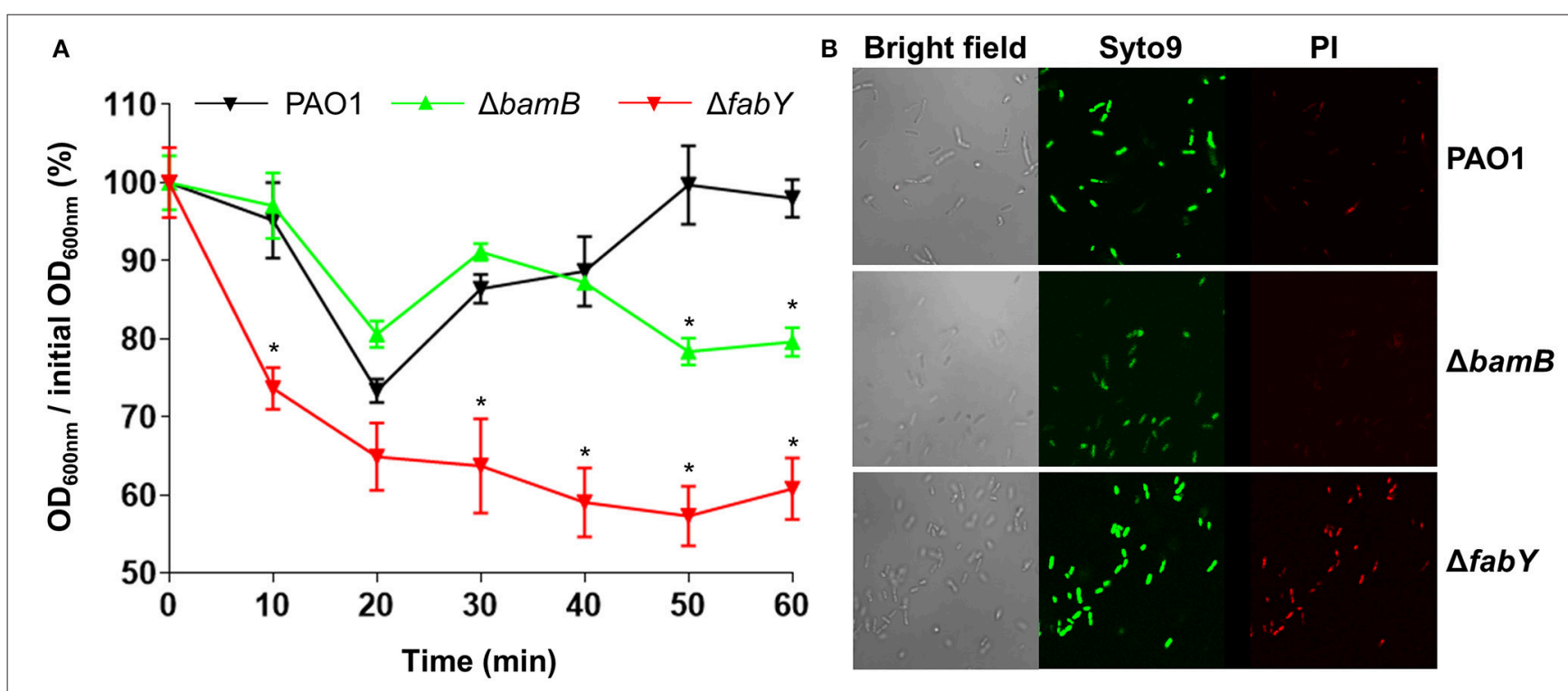

FIGURE 6 | Bacterial membrane integrity tests of two lysozyme-sensitive mutants. (A) Bacterial cells resuspended in distilled water were monitored for their changes in $\mathrm{OD}_{600}$ values at $10 \mathrm{~min}$ intervals for $1 \mathrm{hr}$. Experiments were performed in triplicate, and the mean $\pm \mathrm{SD}$ values are displayed. ${ }^{\star} P<0.05$ vs. OD 600 of $\mathrm{PAO} 1$ at each time point. (B) Each bacterial culture was stained with Syto9 and propidium iodide and visualized by confocal laser scanning microscope (CLSM) as described in Section Materials and Methods.

cell wall-targeting antibiotics and, therefore, (ii) BamB may be an effective target in the elimination of $P$. aeruginosa.

\section{DISCUSSION}

Recalcitrant $P$. aeruginosa infection remains a huge threat to public healthcare. Increasing numbers of metallo- $\beta$-lactamaseproducing clinical isolates have been identified around the world (Corvec et al., 2006; Chin et al., 2011; Jovcic et al., 2011; Piyakul et al., 2012; Van der Bij et al., 2012), and strains exhibiting resistance to colistin, a "last hope" antibiotic, have also been isolated (Lee et al., 2014). Therefore, novel anti-Pseudomonas strategies that do not generate escape mutants are required. We postulate that one such approach is to exploit lysozyme, an antibacterial innate immunoprotein that is abundantly present in the human airway. In this study, we identified PAO1 mutants that became sensitive to lysozyme treatment and provided the clinical perspectives to control $P$. aeruginosa infections. 

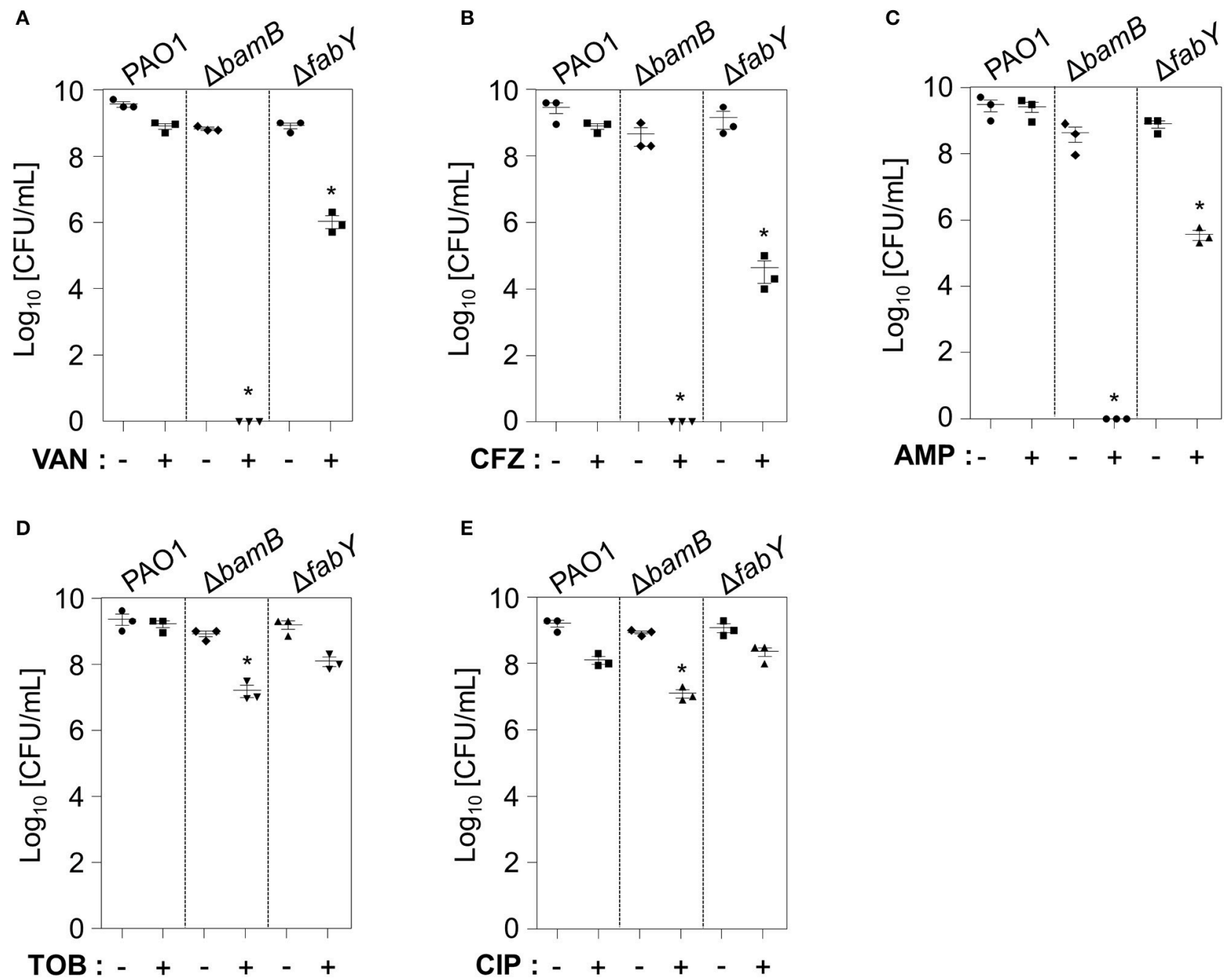

FIGURE 7 | Antibiotic susceptibility of two lysozyme-sensitive PAO1 mutants. PAO1, $\Delta b a m B$, and $\Delta$ fabY mutants were treated in LB for $16 \mathrm{~h}$ with $100 \mu \mathrm{g} / \mathrm{mL}$ Vancomycin (A), $0.5 \mu \mathrm{g} / \mathrm{mL}$ Ceftazidime (B), $25 \mu \mathrm{g} / \mathrm{mL}$ ampicillin (C), $0.2 \mu \mathrm{g} / \mathrm{mL}$ tobramycin (D), or $0.1 \mu \mathrm{g} / \mathrm{mL}$ ciprofloxacin (E). After a16-h incubation, aliquots from each culture were diluted and plated for CFU enumeration. ${ }^{*} P<0.05$ vs. CFU of untreated cultures. Antibiotic concentrations that do not affect PAO1 growth were chosen for analysis.

The outer membrane of Gram-negative bacterial cells is a physical barrier that prevents lysozyme from gaining access to the peptidoglycan layer. EDTA-treated $P$. aeruginosa was found to be sensitive to lysozyme; when grown with $\mathrm{Mg}^{2+}$, the effect of EDTA-mediated increased susceptibility to lysozyme was reversed (Witholt et al., 1976; Ayres et al., 1998). These results suggest that outer membrane integrity is important for lysozyme resistance. In this respect, it is not surprising that lysozymesensitive mutants were also susceptible to antibiotics that target peptidoglycan synthesis. Of particular interest, however, is the extreme sensitivity of the $\triangle$ bamB mutant against cell walltargeting antibiotics (Figure 7). Complete loss of viability was observed in response to treatment with vancomycin, ceftazidime, or ampicillin. These results demonstrate the potential of BamB as a drug target, the inhibition of which could result in efficient elimination of $P$. aeruginosa via a low dose of cell wall-targeting antibiotics. The crystal structure of the BamB indicates that it contains (i) a $\beta$-propeller fold with a central pore region and (ii) protruding loops that mediate its association with BamA (Jansen et al., 2012). BamA homologs in diverse bacterial species are considered essential for viability (Voulhoux et al., 2003; Gentle et al., 2004; Jansen et al., 2012). Given that BamB is an outer membrane protein, we can seek to identify a potential inhibitor that binds to the central pore region of BamB, thereby interfering with its function. Alternatively, the association of BamB with BamA could be targeted for inhibition. Mori and colleagues showed that peptides homologous to a portion of BamA-binding region of BamD can increase the efficacy of antibiotic-mediated killing of $P$. aeruginosa (Mori et al., 2012). It will be important to ask whether these peptides can also potentiate bactericidal 
action of lysozyme in vitro and inside the patient's airway as well.

The $\Delta f a b Y$ mutant produced hypoacylated lipid $\mathrm{A}$ and this change was proposed to be responsible for the increased antibiotic sensitivity (Six et al., 2014). Our result in Figure 6 shows that the $\triangle f a b Y$ mutant is more vulnerable to hypoosmotic stress than $\triangle$ bamB. On the other hand, $\triangle$ bamB mutant cells are far more sensitive than $\Delta f a b Y$ to cell wall-targeting antibiotics (Figure 7). These findings suggest that alterations in fatty acid synthesis or outer membrane protein assembly may lead to unique cell surface changes, which result in distinct consequences. Importantly, both $\Delta b a m B$ and $\Delta f a b Y$ mutant are equally susceptible to the treatment with $1.0 \mathrm{mg} / \mathrm{ml}$ lysozyme (Figure 2). We therefore hypothesize that lysozyme can be widely used in combination with any intervention that targets bacterial cell surface either at the lipid or protein level. We anticipate that the results provided here will stimulate future investigations.

Cerulenin, an anti-fungal agent, inhibits fatty acid biosynthesis by inhibiting $\beta$-ketoacyl-acyl carrier protein synthetase in E. coli (Buttke and Ingram, 1978). PAO1 FabY protein, however, exhibits low homology to the $E$. coli counterpart. Our experiments demonstrated that cerulenin at up to $200 \mu \mathrm{g} / \mathrm{ml}$ does not inhibit $P$. aeruginosa growth (data not shown). To date, a wide range of fatty acid synthesis inhibitors have been identified. These include isoniazid (Chan and Vogel, 2010), triclosan (Wright and Reynolds, 2007), Irgasan (Nishi et al., 2016), Kaempferol (Thors et al., 2008), Quercetin (Zhao et al., 2014a), Apigenin (Brusselmans et al., 2005), 1,2,3,4,6-Penta-O-galloyl- $\beta$-D-glucose (Zhao et al., 2014b), and Epigallocatechin gallage (Brusselmans et al., 2005). Although many of these inhibitors are proved to be effective in eukaryotic cells, these have not been actively tested in bacterial cells. It is worthwhile to examine whether any of these inhibitors can exert

\section{REFERENCES}

Ayres, H. M., Furr, J. R., and Russell, A. D. (1998). Effect of divalent cations on permeabilizer-induced lysozyme lysis of Pseudomonas aeruginosa. Lett. Appl. Microbiol. 27, 372-374. doi: 10.1046/j.1472-765X.1998.00455.x

Beaume, M., Kohler, T., Fontana, T., Tognon, M., Renzoni, A., and van Delden, C. (2015). Metabolic pathways of Pseudomonas aeruginosa involved in competition with respiratory bacterial pathogens. Front. Microbiol. 6:321. doi: 10.3389/fmicb.2015.00321

Bera, A., Biswas, R., Herbert, S., and Gotz, F. (2006). The presence of peptidoglycan O-acetyltransferase in various staphylococcal species correlates with lysozyme resistance and pathogenicity. Infect. Immun. 74, 4598-4604. doi: 10.1128/IAI.00301-06

Berra, L., Sampson, J., and Wiener-Kronish, J. (2010). Pseudomonas aeruginosa: acute lung injury or ventilator-associated pneumonia? Minerva Anestesiol. 76, 824-832. doi: 10.2147/IDR.S50669

Bera, A., Herbert, S., Jakob, A., Vollmer, W., and Gotz, F. (2005). Why are pathogenic staphylococci so lysozyme resistant? The peptidoglycan O-acetyltransferase OatA is the major determinant for lysozyme resistance of Staphylococcus aureus. Mol. Microbiol. 55, 778-787. doi: 10.1111/j.1365-2958.2004.04446.x

Boehnisch, C., Wong, D., Habig, M., Isermann, K., Michiels, N. K., Roeder, T., et al. (2011). Protist-type lysozymes of the nematode Caenorhabditis elegans contribute to resistance against pathogenic Bacillus thuringiensis. PLoS ONE 6:e24619. doi: 10.1371/journal.pone.0024619 synergistic effects on inhibiting $P$. aeruginosa growth, when used together with lysozyme.

In conclusion, this study uncovered a common aspect of two different $P$. aeruginosa mutants with pleiotropic phenotypes. Our results show that interventions that affect fatty acid synthesis or outer membrane protein assembly may facilitate the eradication of $P$. aeruginosa with the aid of physiological concentration of lysozyme. Insights gleaned from this study will be useful in devising new strategies to combat one of the most resistant infections.

\section{AUTHOR CONTRIBUTIONS}

KML and SY conceived, designed, and coordinated the study. KML, KL, JG, IP, JS, JC, and HK performed the experiment and interpreted the data. KML, KL, and SY wrote the manuscript. All the authors participated in discussions of the results and reviewed the final draft.

\section{FUNDING}

This work was supported by grants from the National Research Foundation (NRF) of Korea, funded by the Korean government (2014R1A2A2A01002861, 2014R1A4A1008625, and 2014R1A1A2059520). This work was also made possible by a grant from the Korea Healthcare Technology R\&D Project of the Ministry for Health, Welfare, and Family Affairs (HI15C0694).

\section{SUPPLEMENTARY MATERIAL}

The Supplementary Material for this article can be found online at: http://journal.frontiersin.org/article/10.3389/fcimb. 2017.00059/full\#supplementary-material
Brusselmans, K., Vrolix, R., Verhoeven, G., and Swinnen, J. V. (2005). Induction of cancer cell apoptosis by flavonoids is associated with their ability to inhibit fatty acid synthase activity. J. Biol. Chem. 280, 5636-5645. doi: 10.1074/jbc.M408177200

Buttke, T. M., and Ingram, L. O. (1978). Inhibition of unsaturated fatty acid synthesis in escherichia coli by the antibiotic cerulenin. Biochemistry 17, 5282-5286. doi: 10.1021/bi00617a031

Chan, D. I., and Vogel, H. J. (2010). Current understanding of fatty acid biosynthesis and the acyl carrier protein. Biochem. J. 430, 1-19. doi: 10.1042/BJ20100462

Chin, B. S., Han, S. H., Choi, S. H., Lee, H. S., Jeong, S. J., Choi, H. K., et al. (2011). The characteristics of metallo-beta-lactamase-producing gram-negative bacilli isolated from sputum and urine: a single center experience in Korea. Yonsei Med. J. 52, 351-357. doi: 10.3349/ymj.2011.52.2.351

Corvec, S., Poirel, L., Decousser, J. W., Allouch, P. Y., Drugeon, H., and Nordmann, P. (2006). Emergence of carbapenem-hydrolysing metallo-betalactamase VIM-1 in Pseudomonas aeruginosa isolates in France. Clin. Microbiol. Infect. 12, 941-942. doi: 10.1111/j.1469-0691.2006.1532_1.x

Dajani, R., Zhang, Y., Taft, P. J., Travis, S. M., Starner, T. D., Olsen, A., et al. (2005). Lysozyme secretion by submucosal glands protects the airway from bacterial infection. Am. J. Respir. Cell Mol. Biol. 32, 548-552. doi: 10.1165/rcmb.2005-0059OC

Day, C. A., Marceau-Day, M. L., and Day, D. F. (1993). Increased susceptibility of Pseudomonas aeruginosa to ciprofloxacin in the presence of vancomycin. Antimicrob. Agents Chemother. 37, 2506-2508. doi: 10.1128/AAC.37.11.2506 
Dubin, R. F., Robinson, S. K., and Widdicombe, J. H. (2004). Secretion of lactoferrin and lysozyme by cultures of human airway epithelium. Am. J. Physiol. Lung Cell. Mol. Physiol. 286, L750-L755. doi: 10.1152/ajplung.00326.2003

Duszyk, M. (2001). CFTR and lysozyme secretion in human airway epithelial cells. Pflugers Arch. 443(Suppl. 1), S45-S49. doi: 10.1007/s004240100643

Gentle, I., Gabriel, K., Beech, P., Waller, R., and Lithgow, T. (2004). The Omp85 family of proteins is essential for outer membrane biogenesis in mitochondria and bacteria. J. Cell Biol. 164, 19-24. doi: 10.1083/jcb.200310092

Ghobashy, A. A., and Chiori, C. O. (1984). The combined activity of ampicillin with streptomycin or chloramphenicol against Pseudomonas aeruginosa. Arzneimittelforschung 34, 255-257.

Gi, M., Lee, K. M., Kim, S. C., Yoon, J. H., Yoon, S. S., and Choi, J. Y. (2015). A novel siderophore system is essential for the growth of Pseudomonas aeruginosa in airway mucus. Sci. Rep. 5:14644. doi: 10.1038/srep14644

Go, J., Lee, K. M., Park, Y., and Yoon, S. S. (2014). Extended longevity and robust early-stage development of Caenorhabditis elegans by a soil microbe, Lysinibacillus sphaericus. Environ. Microbiol. Rep. 6, 730-737. doi: 10.1111/1758-2229.12196

Hoff, G. E., Schiotz, P. O., and Paulsen, J. (1974). Tobramycin treatment of pseudomonas aeruginosa infections in cystic fibrosis. Scand. J. Infect. Dis. 6, 333-337. doi: 10.3109/inf.1974.6.issue-4.07

Hughes, W. T., Koblin, B. A., and Rosenstein, B. J. (1982). Lysozyme activity in cystic fibrosis. Pediatr. Res. 16, 874-876. doi: 10.1203/00006450-198210000-00014

Jacquot, J., Tournier, J. M., and Puchelle, E. (1985). In vitro evidence that human airway lysozyme is cleaved and inactivated by Pseudomonas aeruginosa elastase and not by human leukocyte elastase. Infect. Immun. 47, 555-560.

Jansen, K. B., Baker, S. L., and Sousa, M. C. (2012). Crystal structure of BamB from Pseudomonas aeruginosa and functional evaluation of its conserved structural features. PLoS ONE 7:e49749. doi: 10.1371/journal.pone.0049749

Jovcic, B., Lepsanovic, Z., Suljagic, V., Rackov, G., Begovic, J., Topisirovic, L., et al. (2011). Emergence of NDM-1 metallo-beta-lactamase in Pseudomonas aeruginosa clinical isolates from Serbia. Antimicrob. Agents Chemother. 55, 3929-3931. doi: 10.1128/AAC.00226-11

Kim, S., R. M., Seol, S. Y., Yoon, S. S., and Kim, J. (2012). Pseudomonas aeruginosa bacteriophage PA1O requires type IV pili for infection and shows broad bactericidal and biofilm removal activities. Appl. Environ. Microbiol. 78, 6380-6385. doi: 10.1128/A. E. M.00648-12

Lee, J. Y., Na, I. Y., Park, Y. K., and Ko, K. S. (2014). Genomic variations between colistin-susceptible and -resistant Pseudomonas aeruginosa clinical isolates and their effects on colistin resistance. J. Antimicrob. Chemother. 69, 1248-1256. doi: $10.1093 / \mathrm{jac} / \mathrm{dkt} 531$

Lee, K., Lee, K. M., Go, J., Ryu, J. C., Ryu, J. H., and Yoon, S. S. (2016). The ferrichrome receptor A as a new target for Pseudomonas aeruginosa virulence attenuation. FEMS Microbiol. Lett. 363. doi: 10.1093/femsle/fnw104

Lee, K. M., Park Y., Bari, W., Yoon, M. Y., Go, J., Kim, S. C., et al. (2012). Activation of cholera toxin production by anaerobic respiration of trimethylamine N-oxide in Vibrio cholerae. J. Biol. Chem. 287, 39742-39752. doi: $10.1074 /$ jbc.M112.394932

Mallo, G. V., Kurz, C. L., Couillault, C., Pujol, N., Granjeaud, S., Kohara, Y., et al. (2002). Inducible antibacterial defense system in C. elegans. Curr. Biol. 12, 1209-1214. doi: 10.1016/S0960-9822(02)00928-4

Marx, J., Jacquot, J., Berjot, M., Puchelle, E., and Alix, A. J. (1986). Characterization and conformational analysis by Raman spectroscopy of human airway lysozyme. Biochim. Biophys. Acta 870, 488-494. doi: 10.1016/0167-4838(86)90257-8

Merlo, C. A., Boyle, M. P., Diener-West, M., Marshall, B. C., Goss, C. H., and Lechtzin, N. (2007). Incidence and risk factors for multiple antibioticresistant Pseudomonas aeruginosa in cystic fibrosis. Chest 132, 562-568. doi: $10.1378 /$ chest.06-2888

Mori, N., Ishii, Y., Tateda, K., Kimura, S., Kouyama, Y., Inoko, H., et al. (2012). A peptide based on homologous sequences of the beta-barrel assembly machinery component BamD potentiates antibiotic susceptibility of Pseudomonas aeruginosa. J. Antimicrob. Chemother. 67, 2173-2181. doi: 10.1093/jac/dks174

Newman, J. R., and Fuqua, C. (1999). Broad-host-range expression vectors that carry the-arabinose-inducible Escherichia coli araBAD promoter and the araC regulator. Gene 227, 197-203. doi: 10.1016/S0378-1119(98)00601-5
Nishi, K., S. K., Sawamoto, J., Tokizawa, Y., Iwase, Y., Yumita, N., and Ikeda, T. (2016). Inhibition of fatty acid synthesis induces apoptosis of human pancreatic cancer cells. Anticancer Res. 36, 4655-4660. doi: 10.21873/anticanres.11016

O'Callaghan, H. (1986). Ceftazidime, a broad spectrum cephalosporin with activity against Ps. aeruginosa. J. Hyg. Epidemiol. Microbiol. Immunol. 30, 449-453.

Pankhaniya, R. R., Tamura, M., Allmond, L. R., Moriyama, K., Ajayi, T., WienerKronish, J. P., et al. (2004). Pseudomonas aeruginosa causes acute lung injury via the catalytic activity of the patatin-like phospholipase domain of ExoU. Crit. Care Med. 32, 2293-2299. doi: 10.1097/01.CCM.0000145588.79063.07

Pendleton, J. N., Gorman, S. P., and Gilmore, B. F. (2013). Clinical relevance of the ESKAPE pathogens. Expert Rev. Anti Infect. Ther. 11, 297-308. doi: $10.1586 /$ eri. 13.12

Piyakul, C., Tiyawisutsri, R., and Boonbumrung, K. (2012). Emergence of metallobeta-lactamase IMP-14 and VIM-2 in Pseudomonas aeruginosa clinical isolates from a tertiary-level hospital in Thailand. Epidemiol. Infect. 140, 539-541. doi: $10.1017 /$ S0950268811001294

Roy, C., Foz, A., Segura, C., Tirado, M., Teixell, M., and Teruel, D. (1983). Activity of ciprofloxacin (BAYo 9867) against Pseudomonas aeruginosa and ampicillinresistant Enterobacteriaceae. Infection 11, 326-328. doi: 10.1007/BF01641358

Sagel, S. D., Sontag, M. K., and Accurso, F. J. (2009). Relationship between antimicrobial proteins and airway inflammation and infection in cystic fibrosis. Pediatr. Pulmonol. 44, 402-409. doi: 10.1002/ppul.21028

Sawa, T. (2014). The molecular mechanism of acute lung injury caused by Pseudomonas aeruginosa: from bacterial pathogenesis to host response. J. Intensive Care 2:10. doi: 10.1186/2052-0492-2-10

Simon, R., Priefer, U., and Pühler, A. (1983). A broad host range mobilization system for in vivo genetic engineering: transposon mutagenesis in Gram negative bacteria. Biotechnology 1, 784-791. doi: 10.1038/nbt1183-784

Six, D. A., Yuan, Y., Leeds, J. A., and Meredith, T. C. (2014). Deletion of the betaacetoacetyl synthase FabY in Pseudomonas aeruginosa induces hypoacylation of lipopolysaccharide and increases antimicrobial susceptibility. Antimicrob. Agents Chemother. 58, 153-161. doi: 10.1128/AAC.01804-13

Sorrentino, S., Yakovlev, G. I., and Libonati, M. (1982). Dimerization of deoxyribonuclease I, lysozyme and papain. Effects of ionic strength on enzymic activity. Eur. J. Biochem. 124, 183-189. doi: 10.1111/j.1432-1033.1982.tb05923.x

Thors, L., Belghiti, M., and Fowler, C. J. (2008). Inhibition of fatty acid amide hydrolase by kaempferol and related naturally occurring flavonoids. $\mathrm{Br} . J$. Pharmacol. 155, 244-252. doi: 10.1038/bjp.2008.237

Van der Bij, A. K., Van der Zwan, D., Peirano, G., Severin, J. A., Pitout, J. D., Van Westreenen, M., et al. (2012). Metallo-beta-lactamaseproducing Pseudomonas aeruginosa in the Netherlands: the nationwide emergence of a single sequence type. Clin. Microbiol. Infect. 18, E369-E372. doi: 10.1111/j.1469-0691.2012.03969.x

Vanderwinkel, E., de Pauw, P., Philipp, D., Ten Have, J. P., and Bainter, K. (1995). The human and mammalian $\mathrm{N}$-acetylmuramyl-L-alanine amidase: distribution, action on different bacterial peptidoglycans, and comparison with the human lysozyme activities. Biochem. Mol. Med. 54, 26-32. doi: 10.1006/bmme.1995.1004

Verhamme, I. M., Van Dedem, G. W., and Lauwers, A. R. (1988). Ionicstrength-dependent substrate inhibition of the lysis of Micrococcus luteus by hen egg-white lysozyme. Eur. J. Biochem. 172, 615-620. doi: 10.1111/j.1432-1033.1988.tb13933.x

Voss, J. G. (1964). Lysozyme lysis of gram-negative bacteria without production of spheroplasts. J. Gen. Microbiol. 35, 313-317. doi: 10.1099/00221287-35-2-313

Voulhoux, R., Bos, M. P., Geurtsen, J., Mols, M., and Tommassen, J. (2003). Role of a highly conserved bacterial protein in outer membrane protein assembly. Science 299, 262-265. doi: 10.1126/science.1078973

Warren, G. H., Gray, J., and Bartell, P. (1955). The lysis of Pseudomonas aeruginosa by lysozyme. J. Bacteriol. 70, 614-619.

Witholt, B., Heerikhuizen, H. V., and De Leij, L. (1976). How does lysozyme penetrate through the bacterial outer membrane? Biochim. Biophys. Acta 443, 534-544.

Wright, H. T., and Reynolds, K. A. (2007). Antibacterial targets in fatty acid biosynthesis. Curr. Opin. Microbiol. 10, 447-453. doi: 10.1016/j.mib.2007. 07.001

Yoon, M. Y., Lee, K. M., Park, Y., and Yoon, S. S. (2011). Contribution of cell elongation to the biofilm formation of Pseudomonas aeruginosa during anaerobic respiration. PLOS ONE 6:e16105. doi: 10.1371/journal.pone.0016105 
Yoon, S. S., Coakley, R., Lau, G. W., Lymar, S. V., Gaston, B., Karabulut, A. C., et al. (2006). Anaerobic killing of mucoid Pseudomonas aeruginosa by acidified nitrite derivatives under cystic fibrosis airway conditions. J. Clin. Invest. 116, 436-446. doi: 10.1172/JCI24684

Yuan, Y., Sachdeva, M., Leeds, J. A., and Meredith, T. C. (2012). Fatty acid biosynthesis in Pseudomonas aeruginosa is initiated by the FabY class of beta-ketoacyl acyl carrier protein synthases. J. Bacteriol. 194, 5171-5184. doi: 10.1128/JB.00792-12

Zhao, P., Mao, J. M., Zhang, S. Y., Zhou, Z. Q., Tan, Y., and Zhang, Y. (2014a). Quercetin induces HepG2 cell apoptosis by inhibiting fatty acid biosynthesis. Oncol. Lett. 8, 765-769. doi: 10.3892/ol.201 4.2159

Zhao, W., Wang, Y., Hao, W., Zhao, M., and Peng, S. (2014b). In vitro inhibition of fatty acid synthase by 1,2,3,4,6-penta-O-galloyl-beta-D-glucose plays a vital role in anti-tumour activity. Biochem. Biophys. Res. Commun. 445, 346-351. doi: 10.1016/j.bbrc.2014.01.191

Conflict of Interest Statement: The authors declare that the research was conducted in the absence of any commercial or financial relationships that could be construed as a potential conflict of interest.

Copyright $\odot 2017$ Lee, Lee, Go, Park, Shin, Choi, Kim and Yoon. This is an openaccess article distributed under the terms of the Creative Commons Attribution License (CC BY). The use, distribution or reproduction in other forums is permitted, provided the original author(s) or licensor are credited and that the original publication in this journal is cited, in accordance with accepted academic practice. No use, distribution or reproduction is permitted which does not comply with these terms. 